\title{
Sub-nanometer Double Shearing Heterodyne Interferometry for Profiling Large Scale Planar Surfaces
}

\author{
T.Yokoyama, S.Yokoyama, K.Yoshimori* and T.Araki \\ Dept. of Mechanical Science and Bioengineering, Graduate School of Engineering Science, \\ Osaka University
}

*Dept. of Computer and Information Science, Faculty of Engineering, Iwate University

\begin{abstract}
A novel interferometric method, called a double shearing interferometry, is presented for profiling large-scale quasi-planar surfaces, such as semiconductor wafers, optical flats and x-ray mirrors. The surface profile is measured even under existence of the inclination of a scanning stage. By adopting the common-path optical configuration and the heterodyne detection, the proposed method realizes excellent resolution in sub-nanometer order and enables a robust measurement against unwanted disturbances due to the measurement environments. A height resolution of $0.1 \mathrm{~nm}$ was experimentally achieved. A standard deviation of the analytical surface profiles was found to be $1.3 \mathrm{~nm}$, even when using a conventional screw-lead scanning stage. The measured results show that the surface profile and stage inclination are separately determined. We confirmed resultant profile was well consistent with the one measured with Fizeau interferometer.
\end{abstract}

Keywords: heterodyne interferometer, shearing interferometer, profiler, nanometer measurement

\section{Introduction}

There are strong demands for precision measurement of a surface topography of large-scale industrial products, such as silicon wafers, optical flats, and grazing incidence mirrors for synchrotron radiation. Size of the silicon wafer is currently growing up to $300 \mathrm{~mm}$ in diameter, and length of the grazing incidence mirror reaches up to more than $1 \mathrm{~m}$.

A commonly used optical system to measure the topography of the surfaces is Fizeau 
interferometer. Fizeau interferometer informs a profile difference between a surface under test (SUT) and a reference optical flat (ROF) by analyzing the interference fringe pattern. The Fizeau interferometer can measure the whole SUT simultaneously, however, the size of ROF limits the size of SUT which can be measured. Although various methods of fringe pattern analysis, such as phase shifting method (sometimes called fringe scanning method)[1, 2] and Fourier transform method [3], are employed to increase the measurement resolution, uncertainty in measurement is mainly restricted by that of ROF profile. Fizeau interferometer requires a rigid and stable construction to avoid unwanted affection of mechanical vibrations, because SUT and ROF are independently placed, even if the optical paths of them are aligned common-axially. Aiming to measure the large size specimen with high precision, the instrument equipped with ROF whose diameter is $350 \mathrm{~mm}$ has been developed [4]. Measurement uncertainty was investigated and reported as $\frac{\lambda}{77}$, where $\lambda$ is $633 \mathrm{~nm}[5]$.

For measurements of larger size materials such as the grazing incidence mirror, a long trace profiler (LTP) has been developed [6-10]. This instrument scans a single beam that illuminates SUT and measures a slope profile based on an angle-to-position converting feature of the Fourier transform lens. It is reported recently that a 500mm long mirror was measured with the standard deviation of $4.6 \mathrm{~nm}$ in height under the temperature being controlled within \pm 0.1 degree [8].

The shearing interferometry is one of the methods to resolve the issues inherent in the Fizeau interferometer described above, because it does not require ROF. The shearing interferometer uses laterally shifted two probe beams, and measures a differential of the surface profile from the interference signal of those beams. This type of interferometer can employ the heterodyne detection technique, with which sub-nanometer resolution is easily obtained. Sub-nanometer profilers using heterodyne interferometry were reported [11,12], however, their span of measurement was less than several millimeters. To obtain whole surface profile with 
shearing interferometer, mechanical scanning of SUT is necessary and this causes a problem that the affection of inclination of the scanning stage covers small variations of the surface profile.

In this report, we will present a novel interferometric measurement method named $a$ double shearing heterodyne interferometry. The proposed system is composed of two heterodyne shearing interferometers. Here, SUT is illuminated at three points located equidistantly in a straight line with a pair of shearing interferometers to measure two height differences between adjacent two points. Because contribution of the inclination of the scanning stage to the measured two height differences is regarded as equivalent, this contribution is canceled out by subtracting one height difference from the other. Then, one can obtain the second-order differential and retrieve the profile of SUT. In each shearing interferometer, two probe beams propagate through nearly common optical-path by introducing an anisotropic uniaxial crystal (AUC) as a beam splitter. Because of the common-path configuration, affections of the mechanical vibration and fluctuation in the refractive index caused by air turbulence are effectively reduced. Furthermore, temperature drifts of the two shearing interferometers are also reduced by processing an appropriate subtraction treatment.

Based on such common-path optical configuration and the heterodyne detection, a height resolution of $0.1 \mathrm{~nm}$ was experimentally achieved under the usual environment. The height of the profile was measured with the standard deviation of $1.3 \mathrm{~nm}$ using the screw-lead scanning stage. The system is confirmed to be robust and capable of highly sensitive measurement without requirement of extremely stable circumstances.

\section{Principle of the double shearing heterodyne interferometry}

\subsection{Optical system}

The schematic of the optical system is shown in Fig1. A light source of the system is a frequency-stabilized transversal Zeeman He-Ne laser $(\lambda=633 \mathrm{~nm})$ [13]. Output power is $2 \mathrm{~mW}$ and beam diameter ( $\mathrm{e}^{-2}$ of peak intensity) is $0.8 \mathrm{~mm}$. The output lights are linearly polarized and 
their polarization directions are orthogonal due to the Zeeman effect [14]. The frequency difference between two orthogonal lights caused by the cavity mirror anisotropy [15] is approximately $190 \mathrm{kHz}$.

After passing through a half wave plate (HWP), half portion of the laser lights is reflected at a beam splitter (BS) and the orthogonally polarized fields are combined with a polarizer (PL) and detected with a PIN photo diode (PD). Then, the reference beat signal, whose frequency is $190 \mathrm{kHz}$, is generated at the PD due to the square law of the detector. Transmitted lights through BS are split at the parallel plate into two parallel beams whose spatial separation is equal to the sampling pitch of the measurement, $\Delta \mathrm{X}$, and these parallel beams are transmitted through another BS. Each of them is further split with AUC, into the ordinary ray and the extraordinary ray, which are depicted as $\mathbf{0}$ and $\mathbf{e}$, respectively, in Fig.1. The dimension of AUC is designed so that the walk-off distance between the ordinary ray and the extraordinary ray is equal to $\Delta \mathrm{X}$. The optical axis of $\mathrm{AUC}, N$, is set in the plane including two incident parallel beams, so that two pairs of the ordinary and the extraordinary rays lie in the same plane. The ordinary ray from one input beam and the extraordinary ray from the other input beam are superimposed at the output end of AUC. Therefore, the output of AUC consists of parallel three beams, which illuminate three spots on SUT, $X_{n-1}, X_{n}$ and $X_{n+1}$, located equidistantly in a straight line.

HWP is rotated so that the direction of one of two orthogonal polarizations lies in the plane including $N$ and the incident beams. This realizes the condition that one polarization becomes the ordinary ray and the other polarization becomes the extraordinary ray. Thus, each pair of the ordinary and extraordinary rays forms a heterodyne probe of the shearing interferometer whose frequency difference is $190 \mathrm{kHz}$. The output beams of AUC are reflected at SUT and each pair of the ordinary and the extraordinary rays is superimposed again at the front end of AUC. Then, each superimposed beam contains two frequency components that bear the interferometric information of two adjacent spots on SUT. In this manner, the optical 
configuration forms two heterodyne shearing interferometers, which measure the height differences between $X_{n-1}$ and $X_{n}$, and between $X_{n}$ and $X_{n+1}$.

After reflected at BS, two frequency components of each beam are combined with PL and detected with an avalanche photo diode (APD), producing the electrical beat signal of frequency $190 \mathrm{kHz}$. The phase differences of these beat signals relative to the reference beat signal are measured with two phase-meters (PMA and PMB) and registered to a computer at every $\Delta \mathrm{X}$ movement of the scanning stage.

\subsection{Profile retrieval}

The heights of the sample at the positions $X_{n-1}, X_{n}$ and $X_{n+1}$ are denoted as $Z_{n-1}, Z_{n}$ and $\mathrm{Z}_{\mathrm{n}+1}$, respectively, as shown in Fig.1. The height differences $\Delta \mathrm{Z}_{\mathrm{n}-1}$ and $\Delta \mathrm{Z}_{\mathrm{n}}$ are defined as,

$$
\begin{aligned}
& \Delta Z_{n-1}=Z_{n}-Z_{n-1}, \\
& \Delta Z_{n}=Z_{n+1}-Z_{n} .
\end{aligned}
$$

The measured height differences between $X_{n-1}$ and $X_{n}$ and between $X_{n}$ and $X_{n+1}$ are denoted as $\Delta \mathrm{Z}_{\mathrm{A}}(\mathrm{n})$ and $\Delta \mathrm{Z}_{\mathrm{B}}(\mathrm{n})$, respectively. When the inclination of the scanning stage is $\Delta \mathrm{T}_{\mathrm{n}}$ against $\Delta \mathrm{X}$, the measured height differences include $\Delta \mathrm{T}_{\mathrm{n}}$ in addition to the height differences, $\Delta \mathrm{Z}_{\mathrm{n}-1}$ and $\Delta \mathrm{Z}_{\mathrm{n}}$. Then, $\Delta \mathrm{Z}_{\mathrm{A}}(\mathrm{n})$ and $\Delta \mathrm{Z}_{\mathrm{B}}(\mathrm{n})$ are expressed as,

$$
\begin{aligned}
& \Delta Z_{A}(n)=\Delta Z_{n-1}+\Delta T_{n}, \\
& \Delta Z_{B}(n)=\Delta Z_{n}+\Delta T_{n}+\Delta Z_{e r},
\end{aligned}
$$

where $\Delta Z_{\mathrm{er}}$, appeared in the Eq.(2.2), represents the relative error between two independent interferometers. This error is introduced in the calibrating procedure of the initial phases.

From Eq.(2.1) and Eq.(2.2), recursive formula is obtained as,

$$
\Delta Z_{n}-\Delta Z_{n-1}=\Delta Z_{B}(n)-\Delta Z_{A}(n)-\Delta Z_{e r},
$$

then, $\Delta Z_{\mathrm{n}}$ is expressed by 


$$
\begin{aligned}
\Delta Z_{n} & =\sum_{i=1}^{n}\left(\Delta Z_{i}-\Delta Z_{i-1}\right)+\Delta Z_{0} \\
& =\sum_{i=1}^{n}\left(\Delta Z_{B}(i)-\Delta Z_{A}(i)\right)-n \cdot \Delta Z_{e r}+\Delta Z_{0},
\end{aligned}
$$

The height of the sample, $Z_{n}$, is given from Eq.(1.1) as,

$$
\begin{aligned}
Z_{n} & =Z_{n-1}+\Delta Z_{n-1} \\
& =\sum_{i=1}^{n-1} \Delta Z_{i}+Z_{0}+\Delta Z_{0}
\end{aligned}
$$

Then, $Z_{n}$ is obtained from Eq.(4) and Eq.(5). The final expressions for $Z_{n}$ is given by,

$$
Z_{n}=\sum_{i=1}^{n-1}(n-i)\left(\Delta Z_{B}(i)-\Delta Z_{A}(i)\right)-\frac{(n-1) n}{2} \Delta Z_{e r}+n \cdot \Delta Z_{0}+Z_{0}
$$

The stage inclination $\Delta \mathrm{T}_{\mathrm{n}}$ is obtained from Eq.(2.1) and Eq.(4) as,

$$
\Delta T_{n}=\Delta Z_{A}(n)+\sum_{i=1}^{n-1}\left(\Delta Z_{A}(i)-\Delta Z_{B}(i)\right)+(n-1) \Delta Z_{e r}-\Delta Z_{0}
$$

In Eq.(4) to Eq.(7), $Z_{0}$ is initial height at the point $X_{0}$ and $\Delta Z_{0}$ is initial height difference between $X_{0}$ and $X_{1}$. We note that, for retrieving the profile, $Z_{0}$ and $\Delta Z_{0}$ can be chosen as any values without losing generality, because they are merely origins in height and inclination. We also note that the relative error $\Delta Z_{\text {er }}$ gives the second-order polynomial bias to the profile $Z_{n}$ (see Eq.(6)). It also gives the first-order polynomial bias to the stage inclination $\Delta \mathrm{T}_{\mathrm{n}} \quad$ (see Eq.(7)).

To determine the true value of $\Delta \mathrm{Z}_{\mathrm{er}}$, output signals of the two independent interferometers must be compared using a completely flat plane. However, it is not practical to provide such reference flat. Therefore, instead of the true value of $\Delta \mathrm{Z}_{\mathrm{er}}$, the presumed value of $\Delta \mathrm{Z}_{\mathrm{er}}$, denoted $\Delta \mathrm{Z}_{\text {erp }}$, is introduced to calculate the analytical profile. We determine $\Delta \mathrm{Z}_{\text {erp }}$ under the assumption that the average surface of the profile is flat, by applying the standard linear regression method to Eq.(6), that is, $\Delta Z_{\text {erp }}$ is determined so that the second-order polynomial component of the profile vanishes. Then, this presumed value $\Delta Z_{\text {erp }}$ is used to calculate Eq.(6) and Eq.(7). Consequently, the obtained profile, $Z_{n}$, represents the true profile that involves the third and 
higher order polynomial components, at the expense of losing its original parabolic component. On the other hand, the obtained stage inclination, $\Delta \mathrm{T}_{\mathrm{n}}$, represents the true inclination that involves the second and higher order polynomial components, at the expense of losing its original linear component.

We now summarize the actual procedure to retrieve the sample profile: (1) The initial values of $Z_{0}$ and $\Delta Z_{0}$ are once set zero, then, $\Delta Z_{\text {erp }}$ is determined so that the second-order polynomial component of the profile vanishes. (2) The calculations of Eq.(6) and Eq.(7) are performed using $\Delta \mathrm{Z}_{\mathrm{erp}}$ instead of $\Delta \mathrm{Z}_{\mathrm{er}}$. (3) For convenience of showing the profile, the linear component of the obtained profile, $Z_{n}$, is extracted and subtracted from $Z_{n}$. Resultant $Z_{n}$ profile is shown in the figures in the following sections.

\section{Experiments}

In the experimental system, the sampling pitch $\Delta \mathrm{X}$ was $2.7 \mathrm{~mm}$. A calcite beam displacer with walk-off distance of $2.7 \mathrm{~mm}$ (Melles-Griot 03 PPD001) was used as AUC. A distance between AUC and SUT was about $20 \mathrm{~mm}$. SUT was scanned with the stepping-motor-driven screw-lead stages of 85 mm moving span (SGSP20-85, Sigma Koki co.) or $1200 \mathrm{~mm}$ moving span (SGSP65-1200, Sigma koki co.). Phases of the signals, $\Delta \mathrm{Z}_{\mathrm{A}}(\mathrm{n})$ and $\Delta \mathrm{Z}_{\mathrm{B}}(\mathrm{n})$ were measured with two lock-in-amplifiers (5610B, NF co.). SUT was moved with a speed of $1 \mathrm{~mm} / \mathrm{sec}$ and stopped at every $\Delta \mathrm{X}$ of $2.7 \mathrm{~mm}$ and phase data of the lock-in-amplifiers were obtained with time constant of $10 \mathrm{~ms}$ and registered to a computer. The optical system was set up on a vibration-proof-table and the optical path was covered with plastic sheets to prevent the large air turbulence. There were heat sources such as the laser, the lock-in-amplifiers and the computer in the experimental room, however, the room temperature was not specially controlled. Before the sample profile measurement, the system noise was investigated to evaluate the measurement resolution. The phases of $\Delta \mathrm{Z}_{\mathrm{A}}(\mathrm{n})$ and $\Delta \mathrm{Z}_{\mathrm{B}}(\mathrm{n})$ were recorded in 10 minutes. The scanning stage was not moving during this measurement. The result is shown in Fig.2. The phase fluctuation within a short period was smaller than 0.1 degree, which corresponds to the height 
variation of $0.1 \mathrm{~nm}$. In spite of appreciable air turbulence and residual mechanical vibration in the experimental environment, such the excellent height resolution was achieved because of a nearly common-path configuration in each shearing interferometer. The slow phase drift was also found in Fig.2. The temperature change in the experimental room is considered to cause this drift. As seen in Fig.2, the phase drifts of $\Delta Z_{A}(n)$ and $\Delta Z_{B}(n)$ arise in the same way, both in direction and amount. This is because the optical configuration of the two interferometers is the same and the optical path differences of them are approximately the same. Consequently, the drifts of the two phases are successfully canceled through subtracting procedure in the evaluation of $Z_{n}$ and $T_{n}$ (see Eq.(6) and Eq.(7)).

The measured samples were (1) an aluminum-coated glass plate $[70 \mathrm{~mm} \phi \times 10 \mathrm{~mm}$ (D)], (2) a silicon plate [100 $\mathrm{mm}(\mathrm{L})$ x $50 \mathrm{~mm}$ (W) x $10 \mathrm{~mm}$ (D)] and (3) a silicon block for the X-ray mirror [400 mm (L) x $50 \mathrm{~mm}$ (W) x $30 \mathrm{~mm}$ (D)]. The sample (1) was set on an $85 \mathrm{~mm}$ long stage, while the samples (2) and (3) were set on a $1200 \mathrm{~mm}$ long stage. The scanned lengths of the samples (1) (3) were $60 \mathrm{~mm}, 57 \mathrm{~mm}$ and $380 \mathrm{~mm}$, respectively. The measurement time for one scan was 4 minutes for the samples (1) and (2), and 20 minutes for the sample (3). The obtained profiles of the samples (1) (3) are shown in Fig.3(a), Fig.4 and Fig.5, respectively. In Fig.5, rising part is seen in the right side of the the retrieved curve, however, it is not cumulative error of measurement, because $\Delta \mathrm{Z}_{\mathrm{erp}}$ is given so that the retrieved profile does not have the second-order polynomial component. To evaluate the system reproducibility, the samples (1) and (2) were measured 17 and 4 times, respectively, and resulted standard deviations of $Z_{n}$ as 1.32 $\mathrm{nm}$ and $1.30 \mathrm{~nm}$, respectively. The sample (3) was measured 2 times and the average difference of obtained $\mathrm{Z}_{\mathrm{n}}$ values was $11.4 \mathrm{~nm}$. It is considered that the manner of holding the sample (3) was not appropriate, thus, the sample might have slipped slightly during the stage movement. We are going to optimize the holding mechanism of large samples.

Now, we demonstrate the capability of our system to separate the profile from the stage inclination. For this purpose, the data of the sample (3) before the calculation of Eq.(6) are 
shown in Fig.6. In this figure, $A: \Delta Z_{A}(n), B: \Delta Z_{B}(n)-\Delta Z_{\text {erp }}$, which is compensated $\Delta Z_{B}(n)$ with the presumed error value, $\Delta \mathrm{Z}_{\text {erp }}$, and B-A: $\Delta \mathrm{Z}_{\mathrm{B}}(\mathrm{n})-\Delta \mathrm{Z}_{\mathrm{A}}(\mathrm{n})-\Delta \mathrm{Z}_{\text {erp }}$, are shown. In the values of $\mathrm{A}$ and $\mathrm{B}$, the rugged variations, for example, periodic variations with about $10 \mathrm{~mm}$ and $160 \mathrm{~mm}$ periods, can be seen. These variations are caused mainly by the stage tilt, because they appear in both $\mathrm{A}$ and $\mathrm{B}$ in the same behavior. A small difference between values of A and B (see B-A in this figure) is second derivative of the profile as indicated in Eq.(3). Thus, integrating this value twice according to the Eq.(4) and Eq.(5), the profile of SUT is retrieved as shown in Fig.5. The variation of the stage tilt, $\Delta \mathrm{T}_{\mathrm{n}}$, is also calculated using Eq.(7), separately from the profile of SUT. From the plots shown in Fig.5 and Fig.6, one can find how the system retrieves the profile using small signal extracted from measured signals, which are largely occupied with noise due to the stage inclination.

Next, the proposed method was compared with the Fizeau interferometry. The sample (1) was measured with the Fizeau interferometer whose uncertainty is reported as $\frac{\lambda}{77}[4,5]$. The second-order polynomial component was subtracted from the obtained profile for comparison. The result is shown in Fig.3 (b). In this measurement, difference in reflectivity between the sample and the reference plane was large, resulting in a possible decrease of the signal to noise ratio and the system performance. However, the profile of the sample was obtained with enough reliability. Comparing Fig.3 (a) with Fig.3 (b), the profile obtained with the double shearing interferometer shows well consistent with that obtained with the Fizeau interferometer, although there remain quantative differences of the absolute values. In the proposed system, one of the main causes of the absolute error is an optical misalignment of probes. For example, the misalignment of the parallel plate leads to the situations such that two probe lights illuminating the center spot of the three spots on SUT are not superimposed completely and further, the three spots deviate from the location of a straight line. These possible systematic errors should be investigated in the future and uncertainty should be further 
examined.

As the result of the experiments, the good reproducibility of the proposed system and the good agreement with the Fizeau interferometers are obtained and these indicate that the double shearing interferometry is capable of sub-nanometer profiling of large samples.

\section{Interpolation of height within the probe interval}

The spatial frequency that can be measured with the proposed method is limited by the interval of the probes, $\Delta \mathrm{X}$. The highest spatial frequency of the experimental system corresponds to twice of $\Delta \mathrm{X}$ of $2.7 \mathrm{~mm}$. Although narrower $\Delta \mathrm{X}$ can be obtained by selecting an appropriate size of the crystal and the parallel plate, it is hard to detect two interference lights separately when $\Delta \mathrm{X}$ is less than the beam diameter of $0.8 \mathrm{~mm}$. To realize the narrower $\Delta \mathrm{X}$, an additional improvement of the optical configuration should be done. Instead of this, we show in this paper, an improved algorithm of measurement that expands the measurable spatial frequency without changing the optical configuration.

In the improved algorithm, the intermediate points between the original sampling points are additionally measured and the height information of the intermediate points is interpolated. As shown in Fig.8a, the original sampling points, which are $\Delta \mathrm{X}$ apart, are represented by the sequence of the first subscript $i$ of $X_{i, 0}(i=0,1,2, . . N)$, and the intermediate points between $X_{i, 0}$ and $X_{i+1,0}$ are represented by the sequence of the second subscript $j$ of $X_{i, j}(j=1,2, . . M)$. Here, the probe measures height difference between $\mathrm{X}_{\mathrm{i}-1, \mathrm{j}}$ and $\mathrm{X}_{\mathrm{i}, \mathrm{j}}, \Delta \mathrm{Z}_{\mathrm{A}}(\mathrm{i}, \mathrm{j})$, and that between $\mathrm{X}_{\mathrm{i}, \mathrm{j}}$ and $\mathrm{X}_{\mathrm{i}+1, \mathrm{j}}, \Delta \mathrm{Z}_{\mathrm{B}}(\mathrm{i}, \mathrm{j})$. This measurement is conducted by scanning SUT stepwise by the distance $\delta \mathrm{X}$ between $\mathrm{X}_{\mathrm{i}, \mathrm{j}}$ and $\mathrm{X}_{\mathrm{i}, \mathrm{j}+1}$. The calculation of retrieving the profile is performed for $\Delta \mathrm{Z}_{\mathrm{A}}(\mathrm{i}, \mathrm{j})$ and $\Delta \mathrm{Z}_{\mathrm{B}}(\mathrm{i}, \mathrm{j})$ which have the same $\mathrm{j}$. Resultant profile is specified as $P j$, which consists of sequence of heights, $Z_{i, j}(i=0,1, \ldots, N)$, corresponding to $X_{i, j}(i=0,1, \ldots, N)$. In this way, the original 
profile $P_{0}$ and additional $\mathrm{M}$ profiles $P_{1}, P_{2}, \ldots, P_{M}$ are retrieved.

Retrieved $\mathrm{M}$ profiles are connected to $P_{0}$ by modifying the parameters, $\Delta \mathrm{Z}_{\mathrm{erp}}, \mathrm{Z}_{0}$ and $\Delta \mathrm{Z}_{0}$ of each $P j$. Since the measurement is conducted with the same probe that has the unique relative phase difference, $\Delta \mathrm{Z}_{\mathrm{erp}}$ of $P_{0}$ is employed to other profiles. Parameters $\mathrm{Z}_{0}$ and $\Delta \mathrm{Z}_{0}$ of $P j, \mathrm{Z}_{0, j}$ and $\Delta \mathrm{Z}_{0, \mathrm{j}}$, are determined as following steps: (1) Height at $\mathrm{X}_{\mathrm{i}, \mathrm{j}}$ of $P_{0}, \mathrm{Z}^{(0)} \mathrm{i}, \mathrm{j}$, is calculated as linear-interpolating height between $\mathrm{Z}_{\mathrm{i}, 0}$ and $\mathrm{Z}_{\mathrm{i}+1,0}$, as

$$
Z_{i, j}^{(0)}=\frac{j}{M+1} \cdot Z_{i+1,0}+\left(1-\frac{j}{M+1}\right) \cdot Z_{i, 0}
$$

(2) Modified $Z_{i, j}, Z_{i, j}$, is given as,

$$
Z_{i, j}=Z_{i, j}+i \cdot \Delta Z_{0, j}+Z_{0, j}
$$

$\mathrm{Z}_{\mathrm{i}, \mathrm{j}}$ has been calculated assuming both $\mathrm{Z}_{0, \mathrm{j}}$ and $\Delta \mathrm{Z}_{0, \mathrm{j}}$ are zero.

(3) $Z_{0, j}$ and $\Delta Z_{0, j}$ are determined so that the square sum of difference between $Z^{(0)}{ }_{i, j}$ and $Z_{i, j}$ becomes minimum .

$$
\sum_{i=0}^{N}\left(Z_{i, j}^{(0)}-Z_{i, j}\right)^{2} \rightarrow \text { min. }
$$

This algorithm for the interpolation within the probe interval was examined using computer simulation. The line (A) in Fig.8b shows the assumed surface profile for the simulation, which is a low frequency sinusoidal profile with rugged portions whose width is narrower than the probe interval of $\Delta X$. Both amplitude of the low frequency sinusoidal profile and height of rugged portions are $10 \mathrm{~nm}$. The scan length is $50 \mathrm{x} \Delta \mathrm{X}$ and the number of the intermediate points for the interpolation within $\Delta \mathrm{X}$ is $9(\mathrm{M}=9)$, that is, $\delta \mathrm{X}$ is $0.27 \mathrm{~mm}$. The simulation was performed, providing that the probe beam has Gaussian distribution of intensity. Then, measured height 
difference, $\Delta \mathrm{Z}_{\text {meas }}$, with a single shearing interferometer illuminating the points, $\mathrm{X}$ and $\mathrm{X}+\Delta \mathrm{X}$, is given as,

$$
\begin{aligned}
& \Delta Z_{\text {meas }}(X)=\frac{\lambda \phi_{\text {meas }}(X)}{4 \pi}, \\
& e^{i \phi_{\text {meas }}(X)}=\int_{\infty}^{-\infty} \sqrt{\frac{2}{\pi}} \frac{1}{a} e^{-\frac{2(x-X)^{2}}{a^{2}}+i \frac{4 \pi}{\lambda}(Z(x+\Delta X)-Z(x))} d x,
\end{aligned}
$$

where $\mathrm{Z}(\mathrm{x})$ is the assumed profile and $a$ is radius of the probe beam at $\mathrm{e}^{-2}$ of maximum power. In the experimental system, $a$ is given as $0.4 \mathrm{~mm}$.

Fig. 8 b (B) and Fig. $8 \mathrm{~b}(\mathrm{C})$ show the simulation results without and with the interpolation, respectively. The parabolic component has been eliminated from each profile. The high frequency rugged portions are sensed successfully by the interpolation (see Fig. $8 \mathrm{~b}(\mathrm{C})$ ), while they are lost without interpolation (see Fig.8b(B) ). The sensed rugged portions in Fig. $8 \mathrm{~b}(\mathrm{C})$ appear broader comparing with those of the assumed profile. This is because the beam diameter of the probe is larger than $\delta X$.

The simulation results indicate that the algorithm of the interpolation within the probe interval expands the measurable spatial frequency without changing the optical system. We consider our improved algorithm enhances the capability of the double shearing interferometry.

\section{Discussion}

The height of the sample, $\mathrm{Z}_{\mathrm{n}}$, obtained by the proposed system is slightly different from true height, i.e. height perpendicular to the stage surface. When the inclination angle of stage, $\theta_{\mathrm{n}}$, is given as,

$$
\theta_{n}=\tan ^{-1} \frac{\Delta T_{n}}{\Delta X}
$$

height difference of the sample, $\Delta \mathrm{Z}_{\mathrm{n}}$, is expressed with true height difference, $\Delta \mathrm{H}_{\mathrm{n}}$, as, 


$$
\Delta Z_{n}=\frac{\Delta H_{n}}{\cos \theta_{n}}
$$

By substituting Eq.(13) into Eq.(2.1) and Eq.(2,2), true height $H_{n}$ is expressed in the same way as $\mathrm{Z}_{\mathrm{n}}$, as,

$$
H_{n}=\sum_{i=1}^{n-1}(n-i)\left(\Delta Z_{B}(i)-\Delta Z_{A}(i)\right) \cos \theta_{i}-\sum_{i=1}^{n-1}(n-i) \cos \theta_{i} \Delta Z_{e r}+(n-1) \cdot \Delta H_{0}+H_{0}
$$

When $\theta_{\mathrm{n}}$ is small, Eq.(14) reduces to,

$$
H_{n}=\sum_{i=1}^{n-1}(n-i)\left(\Delta Z_{B}(i)-\Delta Z_{A}(i)\right)\left(1-\frac{\theta_{i}^{2}}{2}\right)-\sum_{i=1}^{n-1}(n-i)\left(1-\frac{\theta_{i}^{2}}{2}\right) \Delta Z_{e r}+(n-1) \cdot \Delta H_{0}+H_{0}
$$

Then, the difference between $Z_{n}$ and $H_{n}$, denoted $\delta Z_{n}$, is obtained as,

$$
\begin{aligned}
\delta Z_{n} & =Z_{n}-H_{n} \\
& =\sum_{i=1}^{n-1}(n-i)\left(\Delta Z_{B}(i)-\Delta Z_{A}(i)-\Delta Z_{e r}\right) \frac{\theta_{i}^{2}}{2},
\end{aligned}
$$

where $\mathrm{Z}_{0}, \Delta \mathrm{Z}_{0}, \mathrm{H}_{0}$ and $\Delta \mathrm{H}_{0}$ are assumed to be zero. The magnitude of $\delta \mathrm{Z}_{\mathrm{n}}$ depends on the profile of SUT, however, $\delta Z_{n}$ is considerably small when each $\theta_{i}$ is small. In the present experiment, overall change of $\Delta T_{N}$ was approximately $300 \mathrm{~nm}$ as is seen from Fig.7, thus, overall change of $\theta_{i}$ is estimated as $1.1 \times 10^{-4}$ radian. In this case, the error between $Z_{n}$ and $H_{n}$ is considered to be negligible.

In the current system, two phases are measured discontinuously at every $\Delta \mathrm{X}$ interval. When the phase changes more than $\pi$ between two consecutive measurements, the actual phase change may not be determined uniquely from the measured phase change. Thus, the change in the inclination of the sample must be less than $\frac{\lambda}{4 \cdot \Delta X}$. This limitation is expanded by monitoring the phase change continuously during the scanning, however, there remains another limitation which arises from limited range of detecting light. Two photo detectors of this system are aligned initially so that each interference beam illuminates at the center of corresponding detector. Diameter of the detector is $2 \mathrm{~mm}$ and the path length from SUT to the detectors is 
approximately $100 \mathrm{~mm}$, thus, overall stage inclination during the measurement should be less than $24 \mathrm{mrad}$.

In the proposed retrieval procedure, the second-order polynomial component is lost from the recovered profile, while the higher order polynomial components are obtained correctly. This arises from the presumption of the relative error value, $\Delta Z_{\mathrm{er}}$, between the phases of two independent shearing interferometers. However, in the fields of flatness measurement, low order polynomial components of the profile are often insignificant and thus, these components are removed by the filtering when purposing the measurement of local profiles or roughness. In the field of silicon wafers, especially for that exceeds $300 \mathrm{~mm}$ in diameter, there is a demand for measurement of nanometer height variations with spatial wavelength of 0.2 to $20 \mathrm{~mm}$. Proposed method meets for such demand, because it is capable to measure long span with high sensitivity in height. As for large silicon mirrors or optical flats, such requirements to measure height variations of specific spatial frequencies are expected in manufacturing process.

Measurement time of the experimental system is quite long, particularly for a large sample, because the stage stops at every sampling pitch for measuring. Such the long time measurement may lead erroneous results due to affection of the environmental change during the measurement, although proposed system is robust for temperature variation owing to the configuration of two common-path interferometers. In addition to this, rapid measurement is strongly required for an industrial use. To decrease measurement time, it is necessary to scan SUT continuously. For the continuous measurement, affection due to stage traveling during phase observation must be taken into consideration and simultaneous acquisition of two phases is indispensable. Since time constant of the lock-in amplifier and the speed of stage scan are $10 \mathrm{~ms}$ and $1 \mathrm{~mm} / \mathrm{s}$ respectively, the traveling distance of stage during the time constant is calculated as 10 micron. In this case, the affection due to the stage traveling during observation is considered to be small enough in comparison with the probe interval $(2.7 \mathrm{~mm})$ and diameter $(0.8 \mathrm{~mm})$. Currently, each phase data is acquired at an independent trigger clock of each lock-in-amplifier 
and acquisition time differs by 0.1 second in the worst case. Thus, synchronization of two clocks will enable the continuous measurement.

Objective of the proposed double shearing interferometry is to measure long samples in the range of hundreds of millimeters or more with sub-nanometer height resolution. We confirmed that our method is feasible for this objective through the experiment as shown in the section 3. We are going to improve the moving stage and the holding mechanism to measure longer samples and investigate uncertainty of the system. On the other hand, there is further requirement for the measurement of large samples with higher spatial frequency, for example, in the case of silicon wafers as described above. In the section 4, we proposed the improved algorithm of the measurement to increase the measurable spatial frequency without change of the optical configuration. Measurement of higher spatial-frequency components of the profile can be attained if we realize a smaller spot size and a narrower spot separation. Authors are now implementing an improved optical configuration of the double shearing interferometry. In the new optical system, each probe beam is focused on SUT with the spot size of several tens of microns as well as the spot separation is narrowed to a few hundreds of microns. We plan to report this system in the future.

\section{Conclusions}

The double shearing heterodyne interferometry has been presented for profiling large-scale quasi-planar surfaces with sub-nanometer resolution. In this method, the profile of SUT can be measured separately from the variation of the inclination of the scanning stage. Since the system is composed of a pair of common-path shearing interferometers, the affections of the mechanical vibration and the temperature change are minimized. Based on this optical configuration and the heterodyne detection, the proposed method realizes sensitivity of sub-nanometer order and a robust measurement against the disturbances in the measurement environment.

We have demonstrated the performances of the proposed system. The height resolution of 
$0.1 \mathrm{~nm}$ was achieved under the usual floor environment. The standard deviation of surface profile with the scan length of $60 \mathrm{~mm}$ was $1.3 \mathrm{~nm}$, even though the use of the common screw-lead scanning stage. The measured results show that the surface profiles are determined separately from the stage inclinations. The obtained profile was confirmed to have good agreement with the profile measured with the Fizeau interferometer.

With these results, we may conclude that the proposed method is promising for measurement of sub-nanometer surface profile.

\section{Acknowledgement}

The comparison measurement in this work was performed with the Fizeau interferometer of National Institute of Advanced Industrial Science and Technology (AIST), with the help of Dr.T.Takatsuji and Dr.H.Matsumoto of AIST. The silicon block measurement was supported by Prof. S.Shimada of Osaka Electro Communication University, Dr. A. Komura and Mr.T. Inoue of Hitachi Zosen Co. and Mr. M. Chikamoto of Dept. of Precision Science \& Technology, Osaka University. Authors are grateful to all of them for encouraging results. 


\section{References}

[1] T.Yatagai and T.Kanou, “Aspheric Surface Testing with Shearing Interferometer Using Fringe Scanning Detection Method”, Opt. Engr., 23, 4, 357-360 (1984)

[2] J.H.Bruning, D.R.Herriott, J.E.Gallagher, D.P.Rosenfeld, A.D.White and D.J.Brangaccio, "Digital wavefront measuring interferometer for testing optical surfaces and lenses", Appl. Opt. 13, 11 (1974) 2693

[3] M.Takeda, H.Ina and S.Kobayashi, "Fourier-transform method of fringe-pattern analysis for computer-based topography and interferometry”, J.Opt.Soc.Am., 72, 1 (1982) 156

[4] T.Takatsuji, N.Ueki, K.Hibino, S.Osawa and T.Kurosawa, "Japanese Ultimate Flatness Interferometer (FUJI) and its preliminary experiment”, Proc. SPIE, (2001) 4401

[5] T.Takatsuji, S.Osawa, Y.Kuriyama and T.Kurosawa, "Stability of the reference flat used in Fizeau interferometer and its contribution to measurement uncertainty", Proc. SPIE, $\mathbf{5 1 9 0}(2003) 431$

[6] K.V.Bieren, "Pencil beam interferometer for aspherical optical surfaces, " Proc. SPIE, 343 (1982) 101

[7] S.Qian, W.Jark and P.Z.Takacs, "The Penta-Prism LTP: a long-trace-profiler with stationary optical head and moving penta-prism,” Rev. Sci. Instrum. 66, (1995) 2562

[8] P.Z.Takacs, E.L.Church, C.J.Bresloff and L.Assoufid, "Improvements in the accuracy and the repeatability of long trace profiler measurements," Appl. Opt. 38, 25 (1999) 5468

[9] Y.Zhao, Z.Li, D.Li, X.Tiqiao and X.Shaojian, ”Principle of $\pi$-phase plate long trace profiler for synchrotron radiation optics," Opt. Commun. 200, 23(2001) 
[10] S.Chhatterjee and Y.P.Kumar, "Long-trace profiler with cyclic optical configuration," Appl. Opt. 41, 28 (2002) 5857

[11] G.E.Sommargren, "Optical heterodyne profilometry", Appl. Opt. 20, 4 (1981) 610

[12] D.Pantzer, J.Politch and L.Ek, "Heterodyne profiling instrument for the angstrom region", Appl. Opt. 25, 22 (1986) 4168

[13] T.Yasui, T.Araki and N.Suzuki, "An Intermittent Frequency Offset Lock of a Transverse Zeeman Laser to an Iodine Stabilized He-Ne Laser", Opt. Rev. 3 (1996), 197-201

[14] R.W.Wood : Physical Optics, (Dover Publishing, Inc., New York,1961) 3 $3_{\text {rd }}$ ed., p668.

[15] N.Umeda, M.Tsukiji and H.Takasaki, "Stabilized transverse Zeeman laser as a new light source for optical measurement”, Appl. Opt. 19(1980), 442 


\section{Figure captions}

Fig.1 Schematic of the double shearing interferometer

HWP: half wave plate, PL: polarizer, BS: beam splitter, PD: PIN photodiode, APD: avalanche photo diode, AUC: anisotropic uniaxial crystal, $N$ : the optical axis of AUC, o and e: the ordinary and extraordinary rays, SUT: surface under test, PM: phase meter

Fig.2 The chart record of phase signals, $\Delta \mathrm{Z}_{\mathrm{A}}(\mathrm{n})$ and $\Delta \mathrm{Z}_{\mathrm{B}}(\mathrm{n})$.

Fig.3 Measured profiles of the aluminum-coated glass plate [70 $\mathrm{mm} \phi$ x $10 \mathrm{~mm}$ (D)].

Fig.3(a) measured with the double shearing interferometer 17 times. The standard deviation is $1.32 \mathrm{~nm}$. Fig.3(b) measured with the Fizeau interferometer.

Fig.4 The profile of the silicon plate $[100 \mathrm{~mm}(\mathrm{~L}) \times 50 \mathrm{~mm}(\mathrm{~W}) \times 10 \mathrm{~mm}$ (D)]. It was measured 4 times. The standard deviation is $1.30 \mathrm{~nm}$.

Fig.5 The profile of the silicon block [400 mm (L) x $50 \mathrm{~mm}(\mathrm{~W}) \times 30 \mathrm{~mm}(\mathrm{D})]$

Fig.6 Measured height differences before the retrieval procedure. The sample is the same silicon block in Fig.5

Fig.7 The variation of the stage inclination, $\Delta \mathrm{T}_{\mathrm{N}}$, calculated from data of Fig.6.

Fig.8 Simulation results of interpolation within probe interval.

Fig.8(a) Schematic of sampling points for interpolation.

Fig.8(b) Simulation results. (A) Assumed profile, (B) Simulated profile without interpolation and (C) Simulated profile with interpolation. 


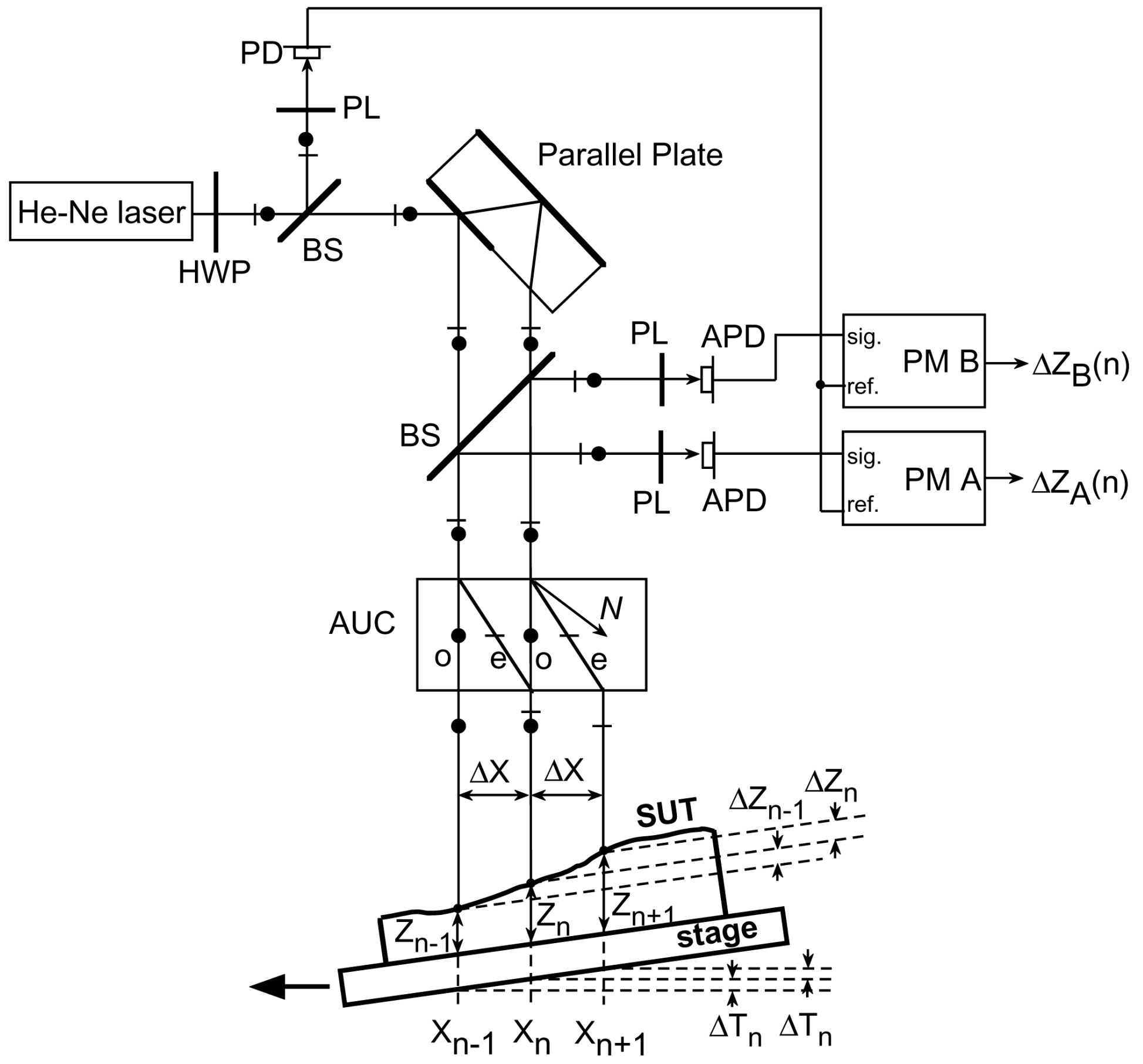

Fig.1 


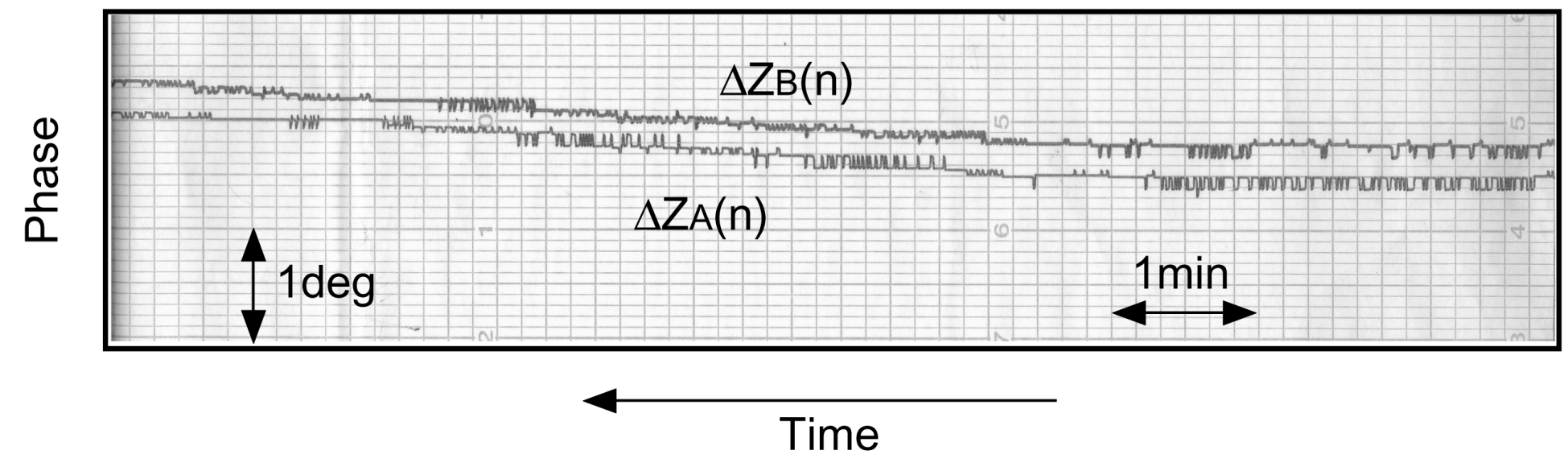

Fig.2 

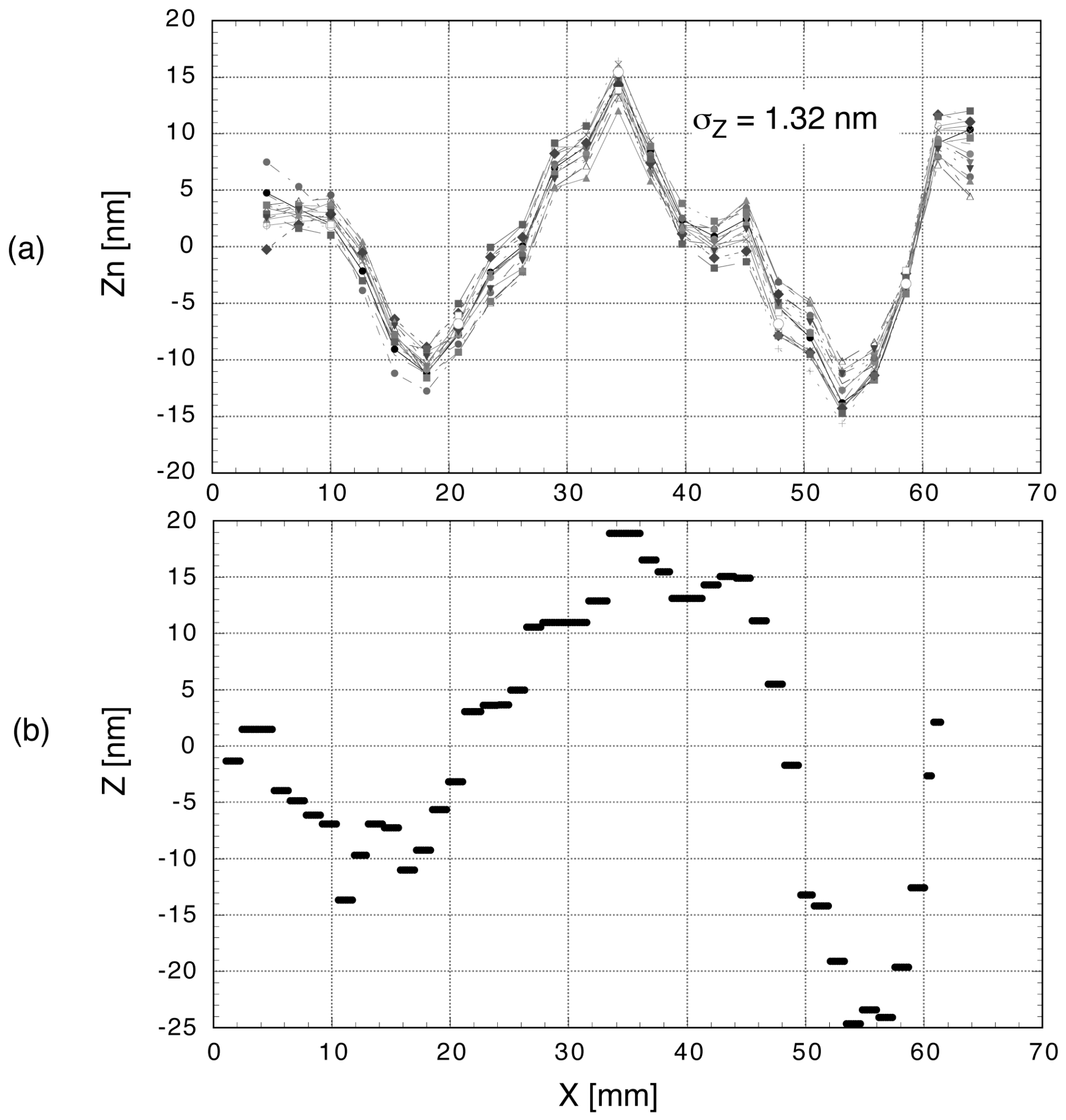

Fig.3 


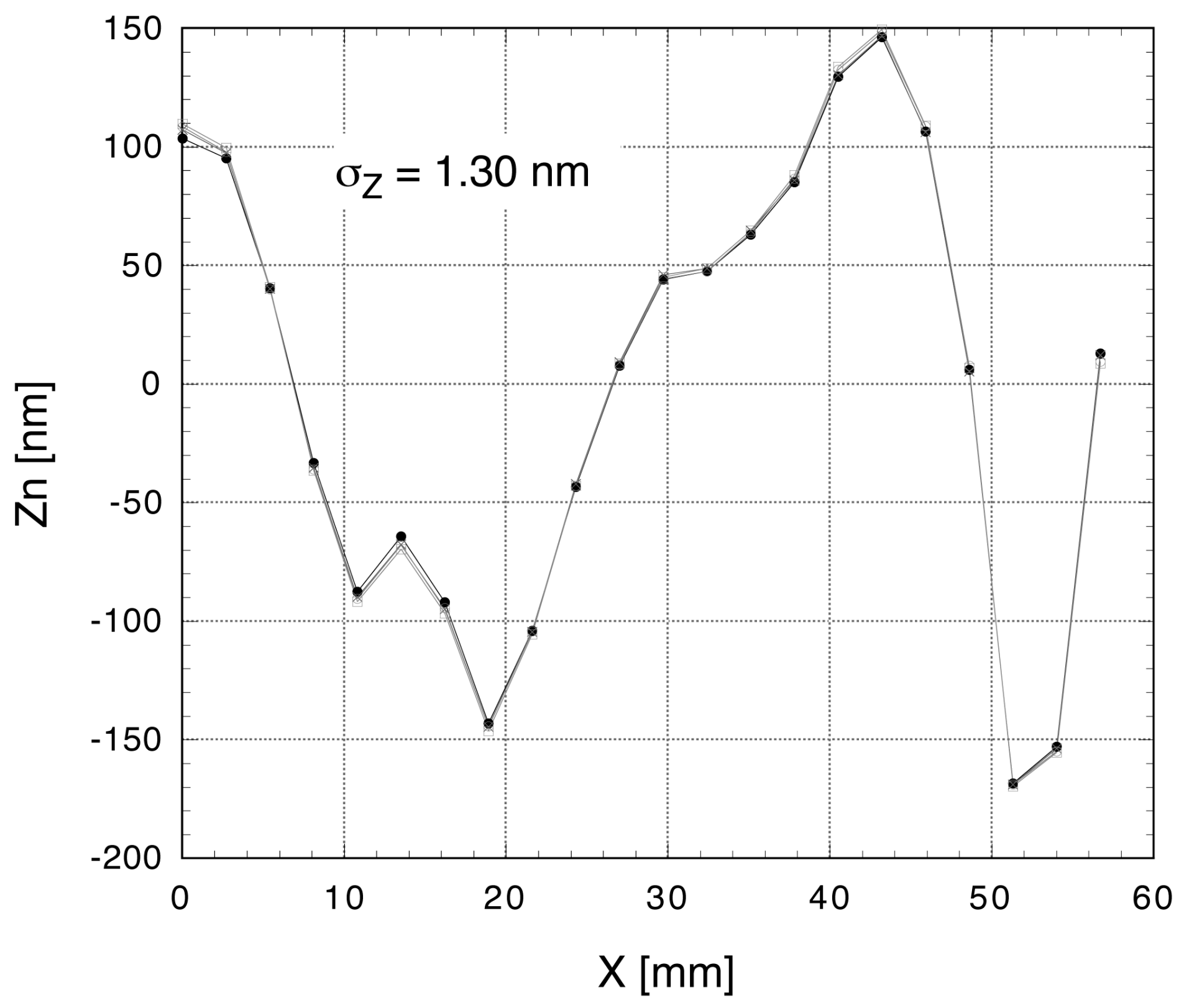

Fig.4 


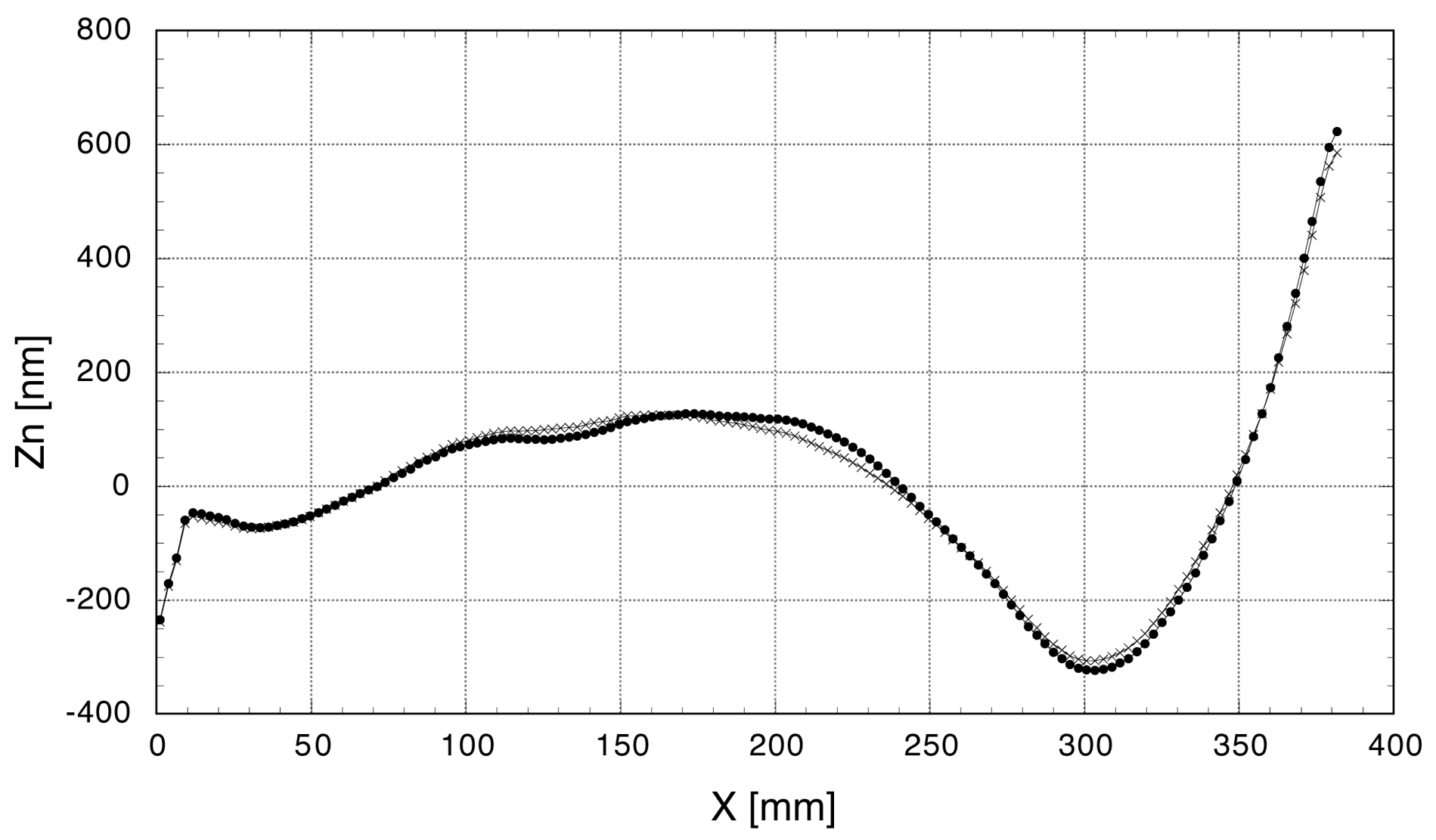

Fig.5 


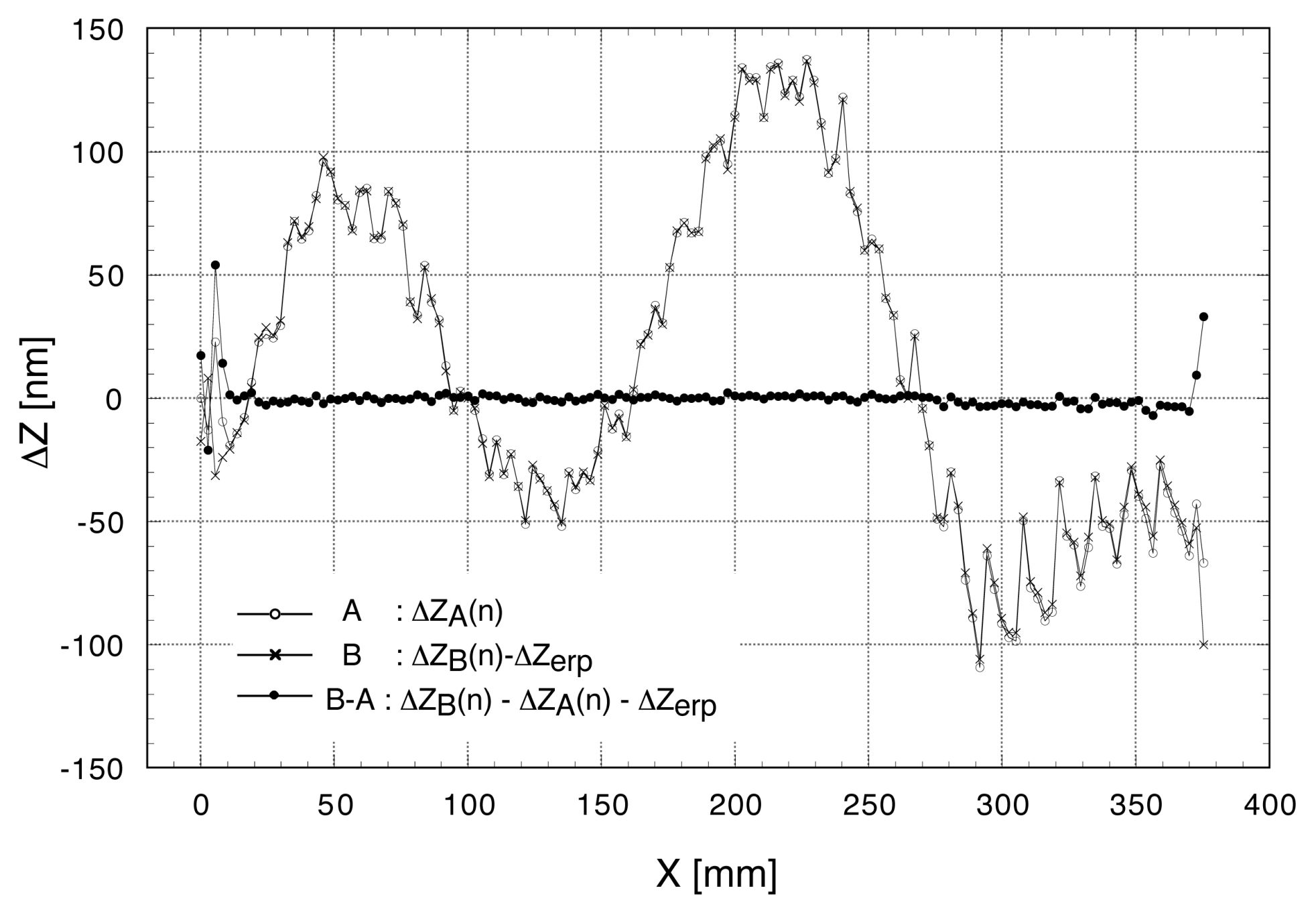

Fig.6 


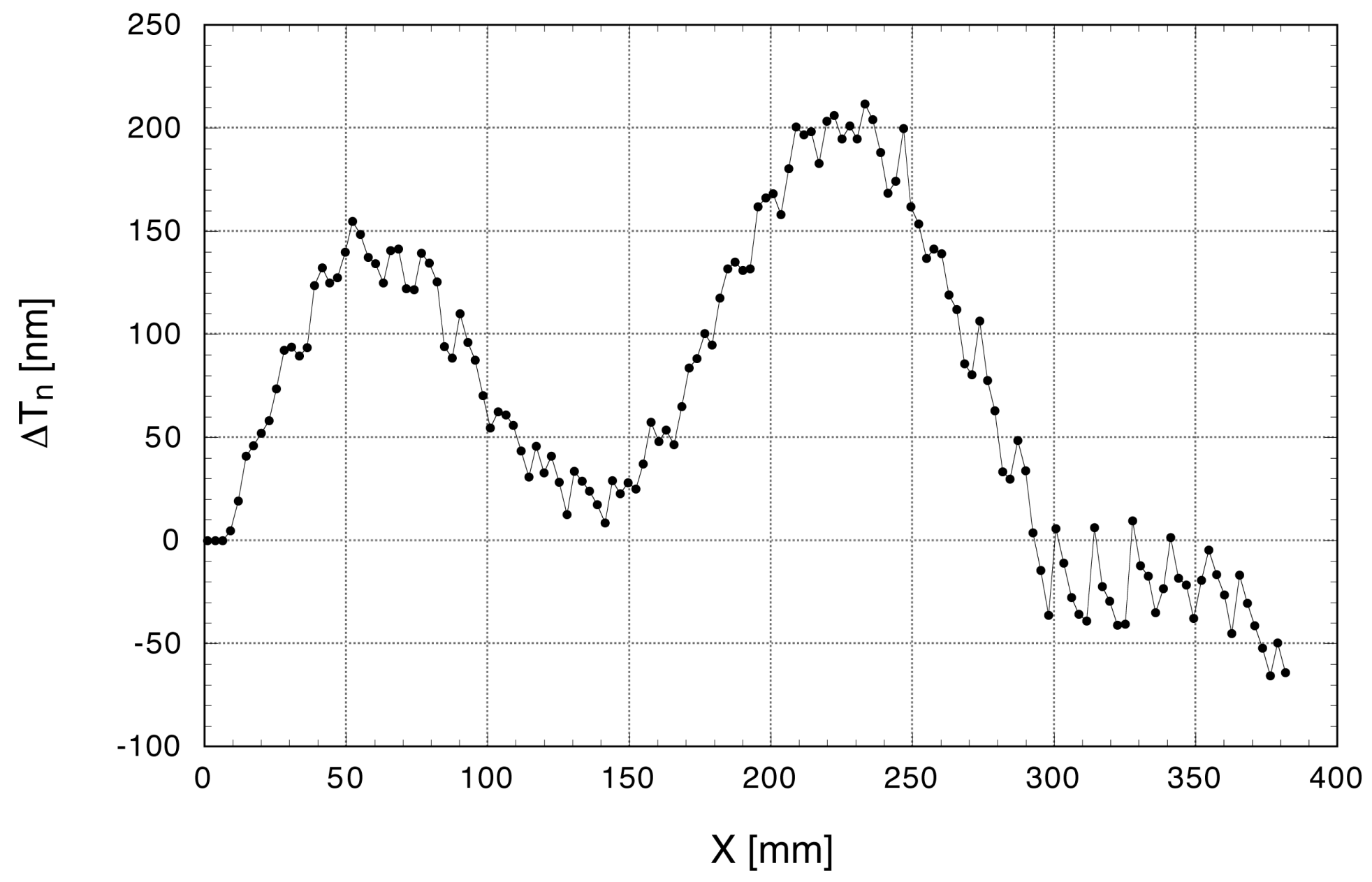

Fig.7 
a.
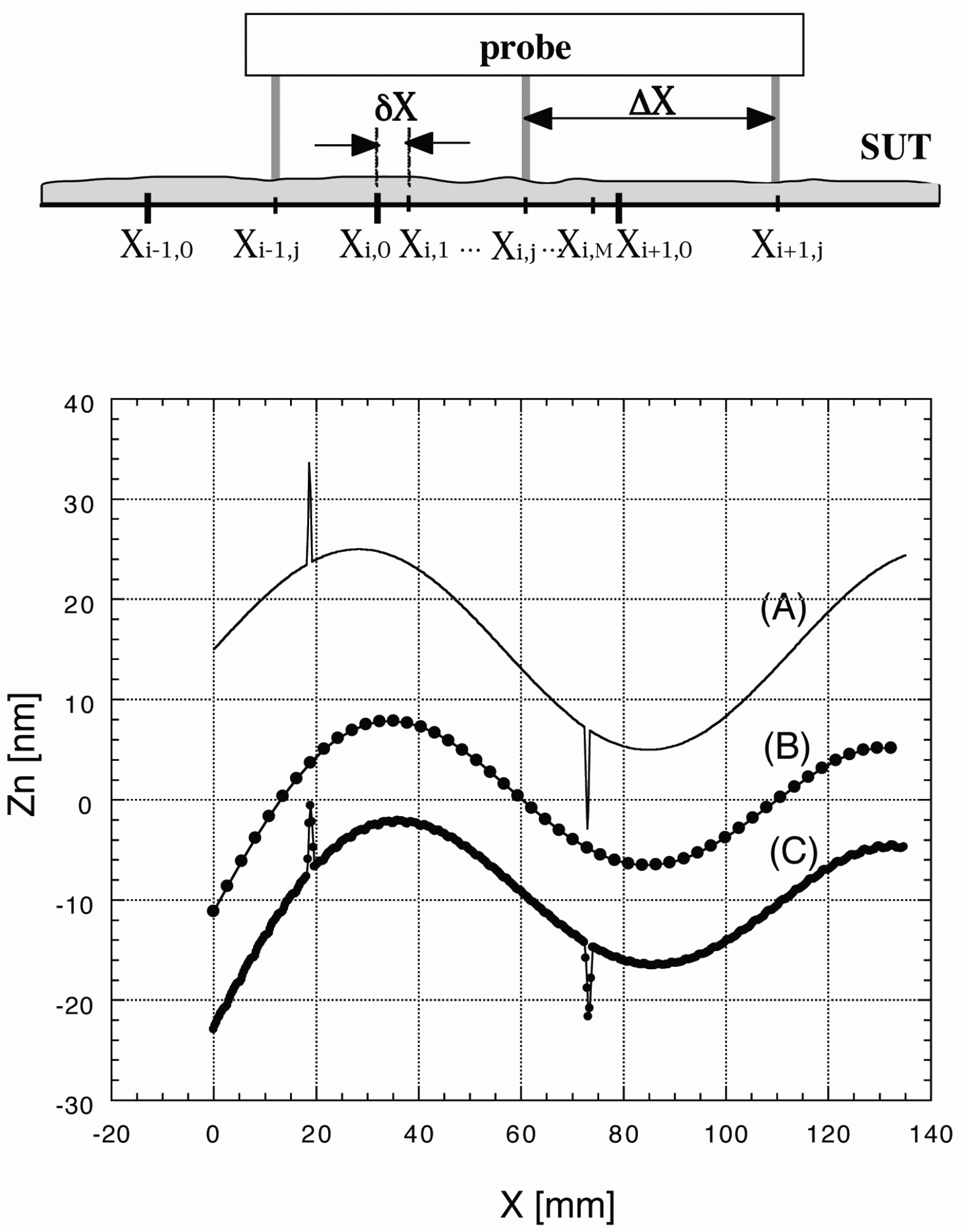

Fig.8 